\title{
POWER SERIES EXPANSIONS OF THE VELOCITY POTENTIAL IN COMPRESSIBLE FLOW*
}

\author{
BY \\ G. K. BATCHELOR \\ Division of Aeronautics, Council for Scientific and Industrial Research, Australia
}

1. The equation for compressible flow. The equation describing the two-dimensional irrotational flow of a compressible fluid is

$$
\frac{\partial^{2} \varphi}{\partial r^{2}}+\frac{1}{r} \frac{\partial \varphi}{\partial r}+\frac{1}{r^{2}} \frac{\partial^{2} \varphi}{\partial \theta^{2}}=\frac{1}{2} M\left[\frac{\partial \varphi}{\partial r} \frac{\partial q^{2}}{\partial r}+\frac{\partial \varphi}{r \partial \theta} \frac{\partial q^{2}}{r \partial \theta}\right]\left[1-\frac{1}{2}(r-1)\left(q^{2}-1\right)\right]^{-1},
$$

where $r, \theta$ are polar coordinates, $M$ is the Mach number of the flow at some distance from the origin where the velocity is assumed to be uniform, $\gamma$ is the ratio of specific heats of the fluid and $\varphi, q$ are the velocity potential and velocity, respectively, at the point $r, \theta$. All distances are referred to some typical dimension in the field, and velocities are referred to the speed of the uniform stream. The boundary conditions to be imposed on $\varphi$ are (1) $\partial \varphi / \partial r \rightarrow \cos \theta$ as $r \rightarrow \infty$, where $\theta$ is measured from the direction of the uniform stream, and (2) the normal derivative of $\varphi$ equals zero at the boundary of a fixed obstacle in the stream.

Many attempts have been made to solve this equation analytically, but none has met with complete success. Most of the progress has been through some linearizing process, such as writing $\varphi$ as a power series in $M^{2}$, the non-linear terms on the right hand side of (1.1) then being of higher order in $M^{2}$ than the linear terms on the left. Again, a transformation to new independent variables, the magnitude and direction of the local fluid velocity, gives a linear equation for $\varphi$. In this paper, use is made of an expansion of $\varphi$ as a power series in $r^{-1}$ and it is shown that the equation for each of the coefficients in the series is linear. Some light is thrown on the form of the expression for $\varphi$ which may be expected in the two cases of zero and finite circulation about the obstacle.

2. Case of zero circulation. For the case of flow past an obstacle about which there is no circulation we make the assumption that $\varphi$ can be written in the following form:

$$
\varphi=r \cos \theta+r^{-1} f_{1}(\theta)+r^{-2} f_{2}(\theta)+r^{-3} f_{3}(\theta)+\cdots .
$$

It will be seen later that the term independent of $\theta$ reduces to a constant when the circulation is zero, and thus may be ignored. Whether or not this expansion for $\varphi$ is valid will be apparent from the subsequent solutions which are obtained for the functions $f_{n}(\theta)$. Invalidity of the assumption (2.1) may be made manifest either as a lack of convergence of the power series in $r^{-1}$, or as a lack of periodicity in $\theta$ of any of the $f_{n}(\theta)$. In the former case, the series could presumably be made convergent by choosing $r$ large enough, so that any conclusions would apply only to regions far from the origin. If, as seems probable, there is no specially favoured region, we may regard the expansion (2.1) as valid generally provided that the resulting equations for the $f_{n}(\theta)$ give periodic solutions.

* Received May 15, 1944. 
The expression for $q^{2}$ corresponding to (2.1) is

$$
\begin{aligned}
q^{2}= & 1+r^{-2}\left(-2 f_{1} \cos \theta-2 f_{1}^{\prime} \sin \theta\right)+\cdots \\
& +r^{-n-1}\left(-2 n f_{n} \cos \theta-2 f_{n}^{\prime} \sin \theta+\cdots\right)+\cdots
\end{aligned}
$$

where dashes denote differentiation with respect to $\theta$, and, in the general coefficient, only terms involving $f_{n}$ have been retained. Substituting both (2.1) and (2.2) in the equation (1.1) and equating coefficients of $r^{-n-2}$ we obtain, after some reduction,

$$
\begin{array}{r}
f_{n}^{\prime \prime}\left(1-M^{2} \sin ^{2} \theta\right)-f_{n}^{\prime}(n+1) M^{2} \sin 2 \theta+f_{n}\left[n^{2}+n M^{2}-\left(n^{2}+2 n\right) M^{2} \cos ^{2} \theta\right] \\
=M^{2} E_{n}\left(f_{n-2}, f_{n-3}, \cdots, f_{1}, \theta\right),
\end{array}
$$

where $E_{n}$ is an undetermined function.

The equation (2.3) may be normalized by writing

which gives for $h_{n}(\theta)$

$$
f_{n}(\theta)=\frac{h_{n}(\theta)}{\left(1-M^{2} \sin ^{2} \theta\right)^{\frac{1}{(n+1)}}}
$$

$$
h_{n}^{\prime \prime}+h_{n}\left\{1+\frac{\left(n^{2}-1\right)\left(1-M^{2}\right)}{\left(1-M^{2} \sin ^{2} \theta\right)^{2}}\right\}=M^{2} E_{n}\left(1-M^{2} \sin ^{2} \theta\right)^{\frac{1(n+1)}{}} .
$$

The homogeneous equation may also be written in the form

where

$$
h_{n}^{\prime}+h_{n}\left(1+u_{0}+\sum_{p=1}^{\infty} 2 u_{p} \cos 2 p \theta\right)=0
$$

$$
u_{p}=\left(n^{2}-1\right)\left(1-M^{2}\right)(-1)^{p} \sum_{m=p}^{\infty} \frac{(2 m) !(m+1)}{(m-p) !(m+p) !}\left(\frac{M}{2}\right)^{2 m} .
$$

The expression for $u_{p}$ is convergent provided $M^{2}<1$ and $\sum u_{p}$ is absolutely convergent for the same condition. The equation (2.6) will be recognized as being identical with Hill's equation which arises in a determination of the motion of the lunar perigee. ${ }^{1}$ As is already known, ${ }^{2}$ Hill's equation may be solved by the assumption of a series expansion of $h_{n}$; for instance, we may write

$$
h_{n}=e^{\mu \theta} \sum_{q=-\infty}^{\infty} b_{q} e^{2 q i \theta}
$$

and determine the constants $b_{q}$, and $\mu$, which governs the periodicity of the solution. However, the method is tedious and we shall find it more expedient to derive a solution in powers of $M^{2}$ from the equation (2.3).

We may also note that if only terms of order $M^{0}$ and $M^{2}$ are retained in (2.6), the homogeneous equation for $h_{n}$ degenerates to Mathieu's equation, viz.,

$$
h_{n}^{\prime}+h_{n}\left[n^{2}-M^{2}\left(n^{2}-1\right) \cos 2 \theta\right]=0 .
$$

It is known that Mathieu's equation yields a periodic solution under certain condi-

1 G. W. Hill, Acta. Math., 8, 1-36 (1886). p. 413.

${ }^{2}$ See for example, Whittaker and Watson, Modern analysis, Camb. Univ. Press, Cambridge, 1940, 
tions; a proof that equation (2.9) gives a periodic solution (with consistent approximation) is demonstrated in the Appendix.

3. Periodicity of $f_{n}$. Consider first the homogeneous equation for $f_{n}$, viz.,

$$
f_{n}^{\prime \prime}\left(1-M^{2} \sin ^{2} \theta\right)-f_{n}^{\prime}(n+1) M^{2} \sin 2 \theta+f_{n}\left[n^{2}+n M^{2}-\left(n^{2}+2 n\right) M^{2} \cos ^{2} \theta\right]=0 .
$$

In order to show that this equation gives a periodic solution for $f_{n}$, we write the solution in the form

$$
f_{n}=F_{n, 0}+M^{2} F_{n, 1}+M^{4} F_{n, 2}+\cdots .
$$

Substituting in (3.1) and equating coefficients of powers of $M^{2}$ we obtain a series of equations, each of the same type, for $F_{n, 0}, F_{n, 1}, \cdots$. The typical equation is

$F_{n, m+1}^{\prime \prime}+n^{2} F_{n, m+1}=F_{n, m}^{\prime \prime} \sin ^{2} \theta+F_{n, m}^{\prime}(n+1) \sin 2 \theta-F_{n, m}\left[n-\left(n^{2}+2 n\right) \cos ^{2} \theta\right]$

the solution of which is periodic unless a term $\cos n \theta$ appears on the right-hand side. Suppose that $F_{n, m}$ consists of the sum of a number of cosine terms; if, for instance,

$$
F_{n, m}=C \cos (p \theta+\delta)
$$

then (3.3) becomes

$$
\begin{aligned}
F_{n, m+1}^{\prime \prime} & +n^{2} F_{n, m+1}=C\left\{\frac{1}{4}(p+n)(p+n+2) \cos [(p+2) \theta+\delta]\right. \\
& \left.+\frac{1}{2}\left(n^{2}-p^{2}\right) \cos (p \theta+\delta)+\frac{1}{4}(n-p)(n-p+2) \cos [(p-2) \theta+\delta]\right\} .
\end{aligned}
$$

$F_{n, m+1}$ will thus be unperiodic only if $p=n-2$; the cases $p=n$ and $p=n+2$ do not give finite terms in $\cos n \theta$ on the right of (3.4). Now the value of $F_{n, 0}$ is

$$
A_{n} \cos \left(n \theta+\epsilon_{n}\right) \quad\left(A_{n}, \epsilon_{n} \text { arbitrary }\right) .
$$

$F_{n, 1}$ therefore contains terms in $\cos (n+2) \theta$ and $\cos n \theta$. Similarly, $F_{n, 2}$ will contain terms in $\cos (n+4) \theta, \cos (n+2) \theta$ and $\cos n \theta$. Generally, we see that a term $\cos (n-2) \theta$ cannot arise in any of the $F_{n, m}$. Hence each of the $F_{n, m+1}$ will be periodic, and the solution of (3.1) is periodic.

Unfortunately, a proof that the particular solution of the equation (2.3) for $f_{n}$ is periodic is not as simple, since the function $E_{n}$ is a very complicated one. For both $n=1$ and $n=2, E_{n}=0$ so that the complete solutions of $f_{1}$ and $f_{2}$ are periodic.

However we can show generally that to the order of $M^{2}$, at least, $f_{n}$ is periodic. To this order the denominator of the right hand side of equation (1.1) may be treated as unity. It is also possible to write, in the numerator of this expression,

$$
f_{n}=A_{n} \cos \left(n \theta+\epsilon_{n}\right)
$$

where $A_{n}$ and $\epsilon_{n}$ are constants independent of $M^{2}$. Considering only the terms of $E_{n}$ which can lead to a term in $\cos n \theta$, we find that

$$
\begin{aligned}
E_{n}= & -2 \cos \theta \sum_{p=1}^{n-2} p(n-p)(n-p-1) f_{p} f_{n-p-1}-2 \cos \theta \sum_{1}^{n-2}(n-p) f_{p}^{\prime} f_{n-p-1}^{\prime} \\
& -2 \sin \theta \sum_{1}^{n-2} p(n-p) f_{p} f_{n-p-1}^{\prime}+2 \sin \theta \sum_{1}^{n-2}(n-p)(n-p-1) f_{p}^{\prime} f_{n-p-1} \\
& + \text { unimportant terms. }
\end{aligned}
$$

By the use of (3.5), this becomes 


$$
\begin{aligned}
E_{n}= & -2 \cos \theta \sum_{1}^{n-2} p(n-p)(n-p-1) A_{p} A_{n-p-1} \cos \left[(n-2 p-1) \theta+\epsilon_{n-p-1}-\epsilon_{p}\right] \\
& +2 \sin \theta \sum_{1}^{n-2} p(n-p)(n-p-1) A_{p} A_{n-p-1} \sin \left[(n-2 p-1) \theta+\epsilon_{n-p-1}-\epsilon_{p}\right]+\cdots \\
= & -2 \sum_{1}^{n-2} p(n-p)(n-p-1) A_{p} A_{n-p-1} \cos \left[(n-2 p) \theta+\epsilon_{n-p-1}-\epsilon_{p}\right]+\cdots,
\end{aligned}
$$

showing that no terms in $\cos n \theta$ occur in $E_{n}$. Hence $f_{n}$ is periodic at least as far as the order of $M^{2}$.

By carrying out in detail the iteration process for a solution of (2.3) in powers of $M^{2}$ for $f_{3}$ and $f_{4}$, and also for $f_{5}$ for the case of a doubly symmetrical body (for which $f_{2}=f_{4}=\cdots=0$ ), the author has been able to show that non-periodicity does not arise when terms of the order of $M^{4}$ are considered, nor does it arise when the order of $M^{6}$ in $f_{3}$ is considered. In addition, the solution of equation (1.1) appropriate to flow past a circular cylinder has been found ${ }^{3}$ to be of the form (2.1), at least as far as the order of $M^{6}$.

Hence, although not yet proved, it seems probable that the particular solution to the equation (2.3) for $f_{n}$ is periodic. If this be the case, then the complete solution for $f_{n}$ is periodic and the assumption of a series expansion (2.1) for $\varphi$ may be regarded as valid for the case of zero circulation.

4. Numerical values of the constants in $f_{1}$. In the case $n=1$, the normalized equation (2.5) becomes

which gives

$$
h_{1}^{\prime \prime}+h_{1}=0
$$

$$
f_{1}=\frac{A \cos (\theta+\alpha)}{1-M^{2} \sin ^{2} \theta}
$$

where $A$ and $\alpha$ are arbitrary constants, to be determined by the inner boundary layer condition. This appears to be the only case for which the complete solution for $f_{n}$ is obtainable, and has also been put foreard by Imai. ${ }^{4}$

In general, $A$ and $\alpha$ are functions of $M^{2}$ and we may write, for small values of $M^{2}$,

$$
\begin{aligned}
A & =a_{0}+a_{1} M^{2}+a_{2} M^{4}+\cdots, \\
\alpha & =\alpha_{0}+\alpha_{1} M^{2}+\alpha_{2} M^{4}+\cdots .
\end{aligned}
$$

Expanding $f_{1}$ in powers of $M^{2}$ we then have $f_{1}=a_{0} \cos \left(\theta+\alpha_{0}\right)+M^{2}\left[a_{0} \cos \left(\theta+\alpha_{0}\right) \sin ^{2} \theta+a_{1} \cos \left(\theta+\alpha_{0}\right)-a_{0} \alpha_{1} \sin \left(\theta+\alpha_{0}\right)\right]+\cdots$.

The value of $f_{1}$ correct to the order $M^{2}$ is known for several different obstacle shapes from the work of previous investigators. For instance, Kaplan ${ }^{5}$ has determined the dence $\beta$ to the uniform stream. Comparing his result with (4.5) we find, after some reduction,

${ }^{3}$ I. Imai, On the flow of a compressible fluid past a circular cylinder, II, Proc. Phys.-Math. Soc. Japan, 23, 180 (1941).

I. Imai, loc. cit.

- C. Kaplan, On the use of residue theory for treating the subsonic flow of a compressible fluid, N.A.C.A. Technical Report 728, 1942. 


$$
\begin{gathered}
a_{0}^{2}=1-\frac{1}{2} c^{2} \cos 2 \beta+\frac{1}{16} c^{4}, \quad(4.6) \quad \tan \alpha_{0}=\frac{c^{2} \sin 2 \beta}{4-c^{2} \cos 2 \beta}, \\
a_{1}=\frac{1}{4 a_{0}}\left[\left(-\frac{1}{16} c^{4}-4-16 c^{-4}\right)+\left(\frac{3}{4} c^{2}+20 c^{-2}\right) \cos 2 \beta+\left(\frac{1}{16} c^{4}-3\right) \cos 4 \beta\right] \\
+\frac{1}{4 a_{0}} \log \frac{4+c^{2}}{4-c^{2}}\left[\left(\frac{1}{2} c^{2}+14 c^{-2}+32 c^{-6}\right)+\left(-\frac{1}{82} c^{4}-5-40 c^{-4}\right) \cos 2 \beta\right. \\
\left.+\left(\frac{1}{8} c^{2}+6 c^{-2}\right) \cos 4 \beta\right], \\
\alpha_{1}=\sin 2 \beta\left[\frac{1}{1}+\frac{1}{2 a_{0}^{2}}\left(1-\frac{1}{4} c^{2} \cos 2 \beta\right)+\frac{1}{8}\left(4 c^{-2}-\frac{1}{4} c^{2}\right) \log \frac{4+c^{2}}{4-c^{2}}\right],
\end{gathered}
$$

where $c$ is the distance between the centre and focus of the ellipse, and the unit of length is the radius of the circle into which the ellipse transforms (equal to the mean of the major and minor axes). At zero incidence, these expressions become

$$
\begin{aligned}
a_{0} & =1-\frac{1}{4} c^{2}=\frac{2 t}{1+t}, \\
a_{1} & =\frac{1}{4}\left(4 c^{-2}-1\right)\left(3-4 c^{-2}\right)+\frac{1}{32} c^{2}\left(4 c^{-2}-1\right)^{4} \log \frac{4+c^{2}}{4-c^{2}} \\
& =\frac{t(1-2 t)}{(1-t)^{2}}-\frac{2 t^{4}}{\left(1-t^{2}\right)(1-t)^{2}} \log t, \\
\alpha_{0} & =\alpha_{1}=0,
\end{aligned}
$$

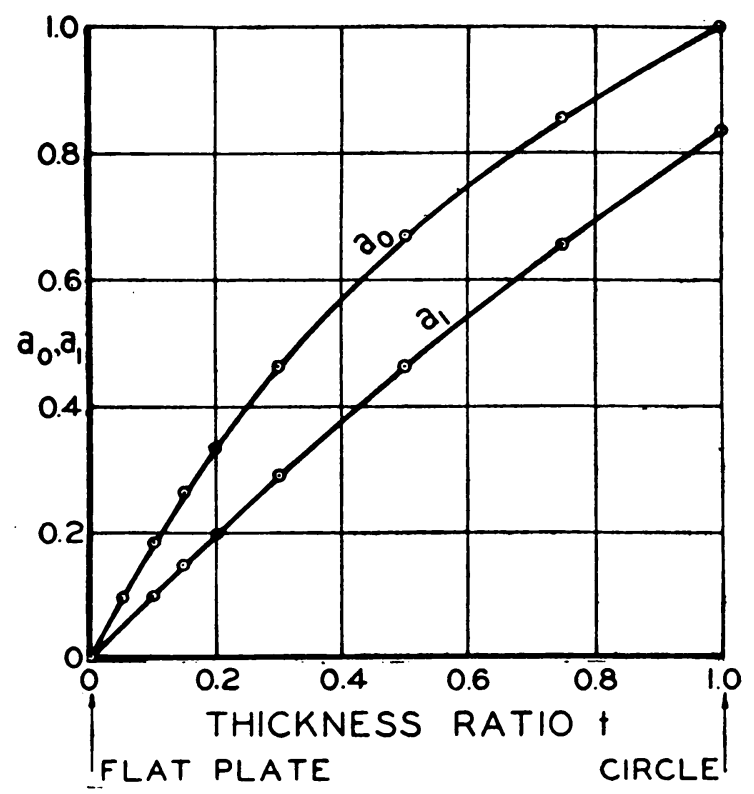

FIG. 1. $a_{0}, a_{1}$, for ellipses ( $0^{\circ}$ incidence).

where $t$ is the ratio of thickness to chord of the ellipse. The expressions (4.10) and (4.11) are shown graphically in Fig. 1. For ellipses of thickness ratio less than $0 \cdot 2$, $a_{1}$ is given accurately by $a_{1}=t$. 
Using results obtained by Tomotika and Umemoto, ${ }^{6}$ it is also possible to derive numerical values for $a_{0}, a_{1}$ corresponding to the flow past a symmetrical Joukowski aerofoil of arbitrary thickness at zero incidence. After considerable reduction, we obtain from a comparison of these authors' formulae with (4.5)

$$
\begin{aligned}
a_{0}= & \frac{1}{4}(1-k)(3+k), \\
a_{1}= & -\frac{1}{64} k^{-8}\left(1-3 k+2 k^{2}+2 k^{3}-3 k^{4}+k^{5}\right)(1+k)^{5} \log \left(1-k^{2}\right) \\
& +\frac{1}{384}\left(-6 k^{-6}-12 k^{-5}+15 k^{-4}+42 k^{-3}-5 k^{-2}-52 k^{-1}+207-170 k\right. \\
& \left.-61 k^{2}+36 k^{3}+10 k^{4}-4 k^{5}\right), \\
\alpha_{0}= & \alpha_{1}=0,
\end{aligned}
$$

where

$$
k=\frac{1-\epsilon}{1+\epsilon}
$$

and $\epsilon$ is the parameter controlling thickness-to-chord ratio in the Joukowski transformation as shown in the table. The unit of length is again the radius of the circle

$$
\begin{array}{llllllllll}
\multicolumn{10}{c}{\text { TABLE-Relation between } \epsilon \text { and } t .} \\
t=0 & 0.03 & 0.05 & 0.07 & 0.10 & 0.15 & 0.20 & 0.30 & 0.50 & \infty \\
t=0 & 0.0378 & 0.0618 & 0.0849 & 0.1179 & 0.1687 & 0.2150 & 0.2958 & 0.4210 & 1
\end{array}
$$

into which the aerofoil transforms. The values of $a_{0}$ and $a_{1}$ are also shown in Fig. 2. Most practical aerofoil shapes have a thickness ratio less than 0.2 and it is evident that $a_{0}$ and $a_{1}$ may be estimated with fair accuracy for these shapes by assuming a linear dependence on $t$. Using the slope appropriate to $t=0$, we have

$$
a_{0}=1.538 t, \quad a_{1}=0.769 t=\frac{1}{2} a_{0} .
$$

From the closeness of the values of $a_{0}$ and $a_{1}$ for an ellipse and a Joukowski aerofoil of the same thickness ratio, it seems reasonable to suppose that $a_{0}$ and $a_{1}$ do not vary greatly with change of shape of the obstacle. These results may then be of use in estimating compressible flow past obstacles of different shapes at points far from the origin.

The same procedure is also possible with the higher coefficients in the series for $\varphi$. For instance, the equation for $f_{2}$ is

$$
f_{2}^{\prime}\left(1-M^{2} \sin ^{2} \theta\right)-3 f_{2}^{\prime} M^{2} \sin 2 \theta+f_{2}\left(4+2 M^{2}-8 M^{2} \cos ^{2} \theta\right)=0
$$

of which the solution, in powers of $M^{2}$, as far as $M^{2}$, is

$$
f_{2}=b_{0} \cos \left(2 \theta+\beta_{0}\right)+M^{2}\left[b_{1} \cos \left(2 \theta+\beta_{1}\right)-\frac{1}{2} b_{0} \cos \left(4 \theta+\beta_{0}\right)\right]+\cdots
$$

where $b_{0}, b_{1}, \beta_{0}, \beta_{1}$ are arbitrary and independent of $M^{2}$. These constants could also be determined from a comparison with known first-order solutions of the compressible

- S. Tomotika and H. Umemoto, On the subsonic flow of a compressible fluid past a symmetrical Joukowski aerofoil, Tokyo Imp. Univ. Aero. Res. Inst., Report 205, 1941.

The expression for the velocity potential for compressible flow given by Tomotika and Umemoto is slightly in error.

In the original symbols the coefficient of $\cos (2 \delta+\alpha)$ in the expression for $\varphi_{1}$ (Eq. 122) is given as $\left(h^{2} / k\right)\left(2+\lambda_{*}+\frac{5}{3} \lambda_{*}^{2}+\frac{2}{3} \lambda_{*}^{3}-8 \lambda_{*}^{2} \log \lambda_{*}\right)$ but should read as $\left(h^{2} / k\right)\left(2+\frac{8}{8} \lambda_{*}^{2}+\frac{2}{3} \lambda_{*}^{4}-8 \lambda_{*}^{2} \log \lambda_{*}\right)$. 
flow past particular obstacles. We may note that for bodies symmetrical about axes parallel and perpendicular to the uniform stream,

$$
b_{0}=b_{1}=\cdots=0 \text {. }
$$

For these doubly-symmetrical bodies, in fact,

$$
f_{2}=f_{4}=\cdots=0 .
$$

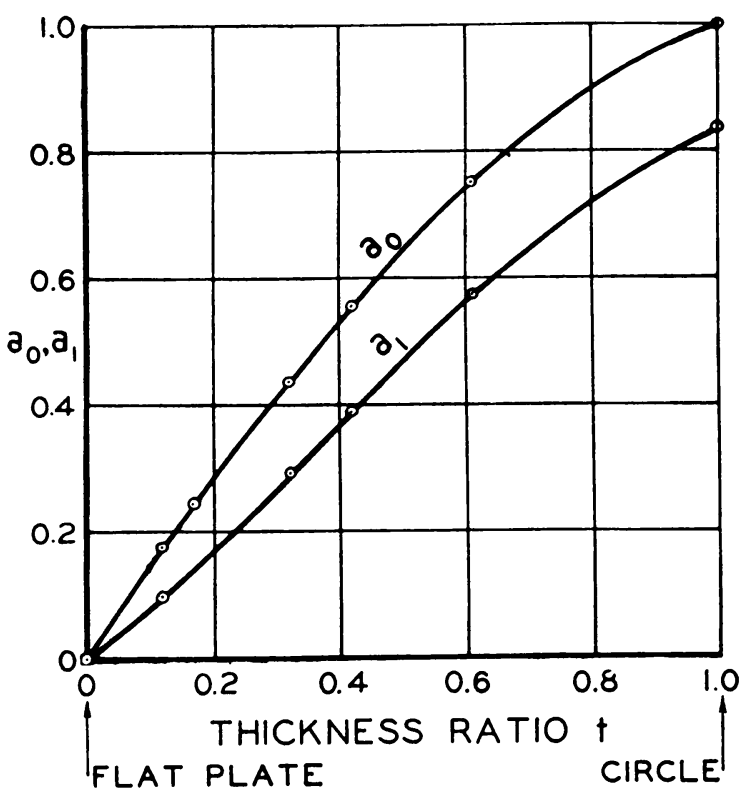

FIG. 2. $a_{0}, a_{1}$ for Joukowski aerofoils $\left(0^{\circ}\right.$ incidence).

5. Case of finite circulation. In the case of flow past a body about which a finite circulation exists, the assumption of a power series expansion for $\varphi$ becomes

$$
\varphi=r \cos \theta+f_{0}(\theta)+r^{-1} f_{1}(\theta)+r^{-2} f_{2}(\theta)+\cdots,
$$

where $f_{0}$ is not periodic, but $f_{1}, f_{2}, \ldots$ must be so. Once again we can take the criterion of validity of this assumption to be the periodicity of the resulting solutions for $f_{1}, f_{2}, \ldots$. Substituting (5.1) in the original equation (1.1) and equating coefficients of $r^{-n-2}$, the equation for $f_{n}$ is obtained as

$f_{n}^{\prime}\left(1-M^{2} \sin ^{2} \theta\right)-f_{n}^{\prime}(n+1) M^{2} \sin 2 \theta+f_{n}\left[n^{2}+n M^{2}-\left(n^{2}+2 n\right) M^{2} \cos ^{2} \theta\right]$

where

$$
=M^{2} E_{n}^{\prime}\left(f_{n-1}, f_{n-2}, \cdots, f_{0}, \theta\right) \text {, }
$$

$$
\begin{aligned}
E_{n}^{\prime}= & E_{n}-2(n+1) \cos \theta f_{0}^{\prime} f_{n-1}^{\prime}-2 \sin \theta f_{0}^{\prime \prime} f_{n-1}^{\prime}-2 \sin \theta f_{0}^{\prime} f_{n-1}^{\prime \prime} \\
& +\frac{1}{2} f_{0}^{\prime} \sum_{p=0}^{n-2}\left[p(n-p-2)\left(f_{p}^{\prime} f_{n-p-2}+f_{p} f_{n-p-2}^{\prime}\right)+f_{p}^{\prime \prime} f_{n-p-2}^{\prime}+f_{p}^{\prime} f_{n-p-2}^{\prime \prime}\right] \\
& -(\gamma-1) f_{0}^{\prime}\left[(n-1)^{2} f_{n-1}+f_{n-1}^{\prime \prime}\right] \\
& +(\gamma-1) f_{0}^{\prime} \sum_{p=0}^{n-2} f_{p}^{\prime}\left[(n-p-2)^{2} f_{n-p-2}+f_{n-p-2}^{\prime \prime}\right] \quad(n>1) .
\end{aligned}
$$


As before, the appearance of a term $\cos n \theta$ in $E_{n}^{\prime}$ at any stage of the solution in powers of $M^{2}$ will indicate that $f_{n}$ is non-periodic and hence that the assumption (5.1) is invalid. We shall in fact show that in this case, non-periodic solutions do arise. However, a more appropriate form for the function $\varphi$ presents itself, and this is shown to lead to a solution which is self-consistent.

Confining attention to the order $M^{2}$, we may write

$$
f_{n}=F_{n, 0}+M^{2} F_{n, 1} \text {. }
$$

$F_{n, 0}$, of course, is the incompressible solution

$$
F_{n, 0}=A_{n} \cos \left(n \theta+\epsilon_{n}\right) .
$$

Substituting (5.4) and (5.5) in (5.2) and equating coefficients of powers of $M^{2}$ we obtain the equation for $F_{n, 1}$ as

$$
\begin{aligned}
F_{n, 1}^{\prime \prime}+n^{2} F_{n, 1}= & A_{n} n(n+1) \cos \left[(n+2) \theta+\epsilon_{n}\right]+A_{n-1}\left\{2 n(n-1) f_{0}^{\prime} \sin \left(n \theta+\epsilon_{n-1}\right)\right. \\
& +2(n-1) f_{0}^{\prime} \sin \left[(n-2) \theta+\epsilon_{n-1}\right] \\
& +2(n-1) f_{0}^{\prime \prime} \sin \theta \sin \left[(n-1) \theta+\epsilon_{n-1}\right] \\
& \left.-\frac{1}{2} f_{0}^{\prime} \sum_{p=0}^{n-2} A_{p} A_{n-p-2}(n-p-2) \sin \left[(n-2 p-2) \theta+\epsilon_{n-p-2}-\epsilon_{p}\right]\right\} \\
& + \text { contribution from } E_{n} .
\end{aligned}
$$

It will be shown later that $f_{\theta}^{\prime \prime}$ is of order $M^{2}$ and may thus be neglected here. As shown previously $E_{n}$ does not give rise to a term $\cos n \theta$ or $\sin n \theta$ in this equation. Hence there is one term on the right of (5.6) which gives a non-periodic particular solution for $F_{n, 1}$; this is

$$
2 n(n-1) f_{0}^{\prime} A_{n-1} \sin \left(n \theta+\epsilon_{n-1}\right)
$$

and the power series expansion (5.1) for $\varphi$ is not valid.

A form for $\varphi$ which will avoid an inconsistent solution is suggested by the relation

$$
\nabla^{2}[\log r \cdot G(r, \theta)]=\frac{2}{r} \frac{\partial G}{\partial r}+\log r \cdot \nabla^{2} G,
$$

where $G$ is an arbitrary function of $r$ and $\theta$, and $\nabla^{2}$ is the Laplacian operator. If we choose $G$ to be a solution of Laplace's equation, in particular,

$$
G=B_{n} \cdot r^{-n} \cos \left(n \theta+\epsilon_{n}^{\prime}\right),
$$

where $B_{n}$ and $\epsilon_{n}$ are arbitrary constants, then

$$
\nabla^{2}(G \log r)=-2 n B_{n} r^{-n-2} \cos \left(n \theta+\epsilon_{n}^{\prime}\right) .
$$

If now, in place of (5.1) we write

$$
\varphi=r \cos \theta+f_{0}+\sum_{n=1}^{\infty} r^{-n}\left[f_{n}+\log r \cdot M^{2} B_{n} \cos \left(n \theta+\epsilon_{n}^{\prime}\right)\right],
$$

the equation (5.6) for $F_{n, 1}$ is unaffected except for the addition to the right-hand side of a term

$$
2 n B_{n} \cos \left(n \theta+\epsilon_{n}^{\prime}\right) .
$$


The particular solution for $F_{n, 1}$ will thus be periodic if we choose

$$
B_{n}=(n-1) f_{0}^{\prime} A_{n-1}, \quad \epsilon_{n}^{\prime}=\epsilon_{n-1}+\pi / 2 \quad(n>1) .
$$

The case of $n=1$ warrants special attention since we cannot write $F_{n-1,0}$ $=A_{n-1} \cos \left[(n-1) \theta+\epsilon_{n-1}\right]$ when $n=1$. When $n=1$, the "dangerous" term on the right of (5.6) is $-2 f_{0}^{\prime 2} \cos \theta$ so that we must choose $B_{1}=f_{0}^{\prime 2}, \epsilon_{1}^{\prime}=0$. Hence,

$$
\begin{aligned}
\sum_{n=1}^{\infty} r^{-n} B_{n} \cos \left(n \theta+\epsilon_{n}^{\prime}\right)= & f_{0}^{\prime 2} \frac{\cos \theta}{r}-f_{0}^{\prime} \sum_{n=2}^{\infty} \frac{(n-1) A_{n-1} \sin \left(n \theta+\epsilon_{n-1}\right)}{r^{n}} \\
=f_{0}^{\prime 2} \frac{\cos \theta}{r}+ & f_{0}^{\prime} \sum_{1}^{\infty}\left\{\cos \theta \frac{\partial}{r \partial \theta}\left[\frac{A_{n} \cos \left(n \theta+\epsilon_{n}\right)}{r^{n}}\right]\right. \\
& \left.+\sin \theta \frac{\partial}{\partial r}\left[\frac{A_{n} \cos \left(n \theta+\epsilon_{n}\right)}{r^{n}}\right]\right\} .
\end{aligned}
$$

But

$$
r \cos \theta+\kappa_{0} \theta+\sum_{n=1}^{\infty} r^{-n} A_{n} \cos \left(n \theta+\epsilon_{n}\right)=\varphi_{0},
$$

where $\varphi_{0}$ is the velocity potential for incompressible flow and $2 \pi \kappa_{0}\left(=2 \pi f_{0}^{\prime}\right.$, neglecting terms in $M^{2}$ ) is the corresponding circulation. Thus

$$
\sum_{1}^{\infty} r^{-n} B_{n} \cos \left(n \theta+\epsilon_{n}^{\prime}\right)=\kappa_{0}\left(\cos \theta \frac{\partial \phi_{0}}{r \partial \theta}+\sin \theta \frac{\partial \phi_{0}}{\partial r}\right)=\kappa_{0} \frac{\partial \phi_{0}}{\partial y},
$$

where $y$ is the space ordinate at right angles to the direction of the free stream.

We have then, that when a finite circulation exists, the expression for $\varphi$, correct to the order $M^{2}$ at least, is

$$
\varphi=r \cos \theta+f_{0}+\sum_{n=1}^{\infty} r^{-n} \cdot f_{n}+M^{2} \kappa_{0} \log r \cdot \frac{\partial \phi_{0}}{\partial y}
$$

and this expression is confirmed by the known results for a circular cylinder. ${ }^{7}$ We may also note, as a matter of convenience, that the expression for $f_{n}$ in (5.16) may be obtained by formally ignoring the log term when substituting in (1.1), and rejecting any non-periodic solutions.

6. Solutions for $f_{0}$ and $f_{1}$. Putting $n=0$ in (5.2) and noting that $E_{0}^{\prime}=0$, we have

$$
f_{0}^{\prime \prime}=\frac{M^{2} \sin 2 \theta}{1-M^{2} \sin ^{2} \theta} f_{0}^{\prime}
$$

integration of which gives

$$
f_{0}^{\prime}=\frac{\kappa}{1-M^{2} \sin ^{2} \theta},
$$

where $\kappa$ is a constant which may depend on $M^{2}$. This equation shows that the circulation about the body in compressible flow is

$$
C=\int_{0}^{2 \pi} f_{0}^{\prime} d \theta=\frac{2 \pi \kappa}{\left(1-M^{2}\right)^{1 / 2}} .
$$

? S. Tomotika and H. Umemoto, loc. cit., Appendix. 
Substituting for $\kappa$ from (6.3) in (6.2) gives $f_{0}^{\prime}$ in terms of the circulation, and an identical equation for the transverse velocity at large $r$ has been found by Glauert ${ }^{8}$ using "small perturbation" theory.

The contribution to the velocity potential is found by integration of (6.2) to be

$$
f_{0}=\kappa\left(1-M^{2}\right)^{-1 / 2} \arctan \left[\left(1-M^{2}\right)^{1 / 2} \tan \theta\right]
$$

or, in terms of the circulation $C$,

$$
f_{0}=\frac{C}{2 \pi} \arctan \left[\left(1-M^{2}\right)^{1 / 2} \tan \theta\right] .
$$

Just as the magnitude of the circulation about a body in incompressible flow is arbitrary, so in compressible flow, the relation betwcen circulation and Mach number is arbitrary. In certain cases an independent criterion for this relation is available, e.g., for sharp-tailed bodies the circulation should be such as to prohibit an infinite velocity at the tail-point for all Mach numbers. For blunt bodies, a convenient criterion is that the circulation shall be the same at all Mach numbers. In this case, we may treat $C$ as a constant and the expansion of $f_{0}$ in powers of $M^{2}$ is

$$
f_{0}=\frac{C}{2 \pi}\left[\theta-\frac{1}{4} M^{2} \sin 2 \theta+M^{4}\left(-\frac{1}{8} \sin 2 \theta+\frac{1}{32} \sin 4 \theta\right)+\cdots\right] .
$$

(Actually this expression for $f_{0}$ has not been proved valid for powers of $M^{2}$ beyond the first. However it seems probable that equation (6.1) for $f_{0}$ will hold whatever the form of the remainder of the expression for $\varphi$. In the case of a circular cylinder with circulation, it is not hard to show that (6.5) is correct to the order $M^{4}$ at least.)

Putting $n=1$ in equation (5.2), we have

$$
f_{1}^{\prime \prime}\left(1-M^{2} \sin ^{2} \theta\right)-2 f_{1}^{\prime} M^{2} \sin 2 \theta+f_{1}\left(1+M^{2}-3 M^{2} \cos ^{2} \theta\right)=0,
$$

the only finite terms in $E_{1}^{\prime}$ being terms which would give rise to non-periodic particular solutions. As shown in section 2, the solution is

$$
f_{1}=\frac{A \cos (\theta+\alpha)}{1-M^{2} \sin ^{2} \alpha}
$$

where we are now justified in using this expression to the order $M^{2}$ only; i.e.,

$$
\begin{aligned}
f_{1}=a_{0} & \cos \left(\theta+\alpha_{0}\right) \\
& +M^{2}\left[a_{0} \cos \left(\theta+\alpha_{0}\right) \sin ^{2} \theta+a_{1} \cos \left(\theta+\alpha_{0}\right)-a_{0} \alpha_{1} \sin \left(\theta+\alpha_{0}\right)\right],
\end{aligned}
$$

the constants having the same meaning as in section 2 but different values owing to the finite circulation.

As in the previous work, it is possible to derive the values of $a_{0}, a_{1}, \alpha_{0}$ and $\alpha_{1}$ by comparison of (6.8) with the known results for certain obstacles. Results are available ${ }^{9}$ for the flow of a compressible fluid about a circular cylinder of unit radius, with constant circulation $C$. Comparison with (6.8) shows that

$$
a_{0}=1, \quad a_{1}=\frac{5}{6}+\left(\frac{C}{2 \pi}\right)^{2},
$$

${ }^{8} \mathrm{H}$. Glauert, The effect of compressibility on the lift on an aerofoil, Proc. Roy. Soc., A 118, 113 (1928).

S. Tomotika and H. Umemoto, loc. cit., Appendix. 
so that the value of $f_{1}$ is not greatly altered by the presence of a small circulation.

We may also note that for a body, symmetrical about axes parallel and perpendicular to the uniform stream

$$
f_{1}^{\prime}(\theta)=f_{1}^{\prime}(\pi-\theta),
$$

giving $\alpha_{0}=\alpha_{1}=0$ and a corresponding result holds for $f_{3}, f_{5}, \cdots$. In the case of even values of $n$, the angle constants in $f_{r}$ are equal to $\pi$.

7. Appendix; Proof that the equation $y^{\prime \prime}+y\left[n^{2}-M^{2}\left(n^{2}-1\right) \cos 2 \theta\right]=0$ gives a periodic solution. The general form of Mathieu's equation ${ }^{10}$ may be taken as

$$
y^{\prime \prime}+y(a+16 q \cos 2 \theta)=0
$$

the solution of which is periodic only if $a$ and $q$ are suitably related. The required relation may be written

$$
a=n^{2}+a_{1} q+a_{2} q^{2}+\cdots,
$$

since $a$ must reduce to the square of an integer when $q$ vanishes. Now, for the particular case of Mathieu's equation with which we are concerned,

$$
a=n^{2}, \quad 16 q=-M^{2}\left(n^{2}-1\right),
$$

and (7.2) will be satisfied to the order of $M^{2}$ if $a_{1}=0$, or, when $n=1$, by any value of $a_{1}$. Thus we need to show that $a_{1}=0$ for the general Mathieu equation.

This may be done by assuming a solution of (7.1) of the form

$$
y=\cos n \theta+q \alpha_{1}(\theta)+q^{2} \alpha_{2}(\theta)+\cdots,
$$

where $\alpha_{1}(\theta), \alpha_{2}(\theta), \cdots$ are periodic functions of $\theta$, independent of $q$. (The proof for solutions which reduce to $\sin n \theta$ when $q=0$ is identical.) Substituting (7.2) and (7.4) in (7.1) and equating coefficients of $q$ we obtain

$$
\alpha_{1}^{\prime \prime}+n^{2} \alpha_{1}+\cos n \theta\left(a_{1}+16 \cos 2 \theta\right)=0,
$$

i.e.,

$$
\alpha_{1}^{\prime \prime}+n^{2} \alpha_{1}^{\prime \prime}=8 \cos (n+2) \theta+a_{1} \cos n \theta+8 \cos (n-2) \theta
$$

giving $a_{1}=0,(n \neq 1)$ since $\alpha_{1}$ is periodic. For the particular case $n=1$ we have instead $a_{1}=-8$, but in this case we no longer require $a_{1}$ to be zero.

Further coefficients $a_{2}, a_{3}, \cdots$ and the functions $\alpha_{1}, \alpha_{2}, \cdots$ may be determined by equating higher powers of $q$; the general Mathieu function may thus be constructed if desired.

${ }^{10}$ Whittaker and Watson, loc. cit., p. 409. 